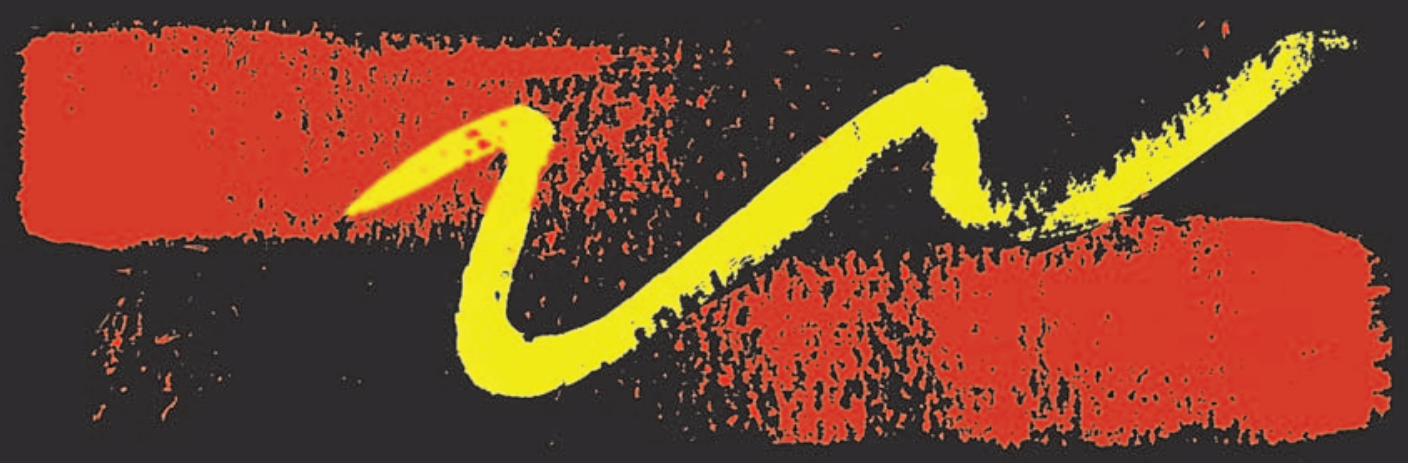

Vol. XXV (1), 2007
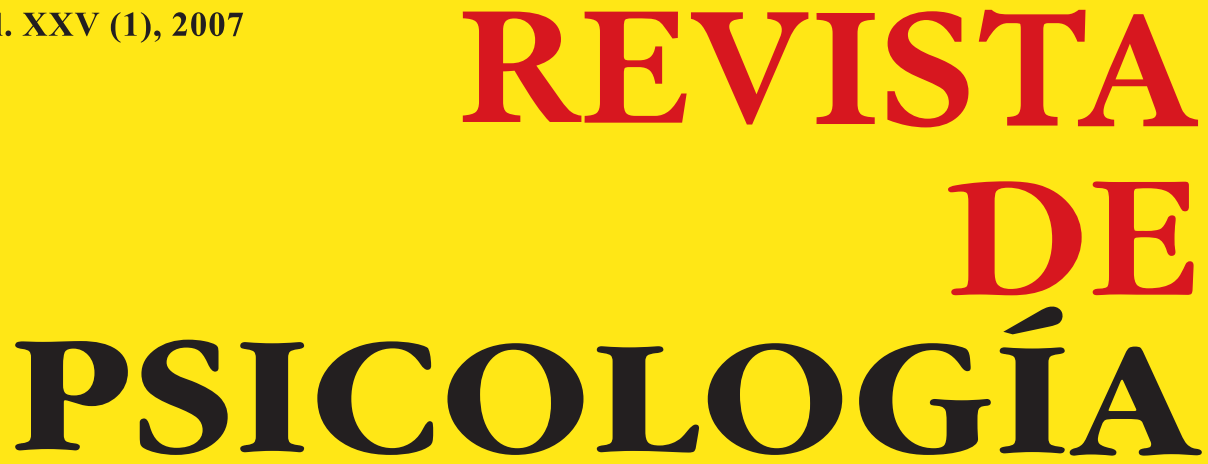

Sandra Castañeda

Anne Marie Costalat-Founeau

Daniel González

Jorge Haddad

Dirk Hermans

Carlos Iberico

Pierina Traverso

Debora Vansteenwegen

César Varela

Bram Vervliet 


\section{REVISTA DE PSICOLOGÍA \\ Vol. XXV (1), 2007}

\section{CONTENIDO}

\section{ARTÍCULOS}

Daniel González Lomelí, Sandra Castañeda Figueiras y César Varela Romero. Proceso de respuesta a examen de egreso en contabilidad: validación de constructo

Jorge Haddad Barthelemy. Experiencias y consideraciones en la conformación de perfiles de competencias

Pierina Traverso $K$. Dos madres adolescentes, dos vínculos: ¿qué marca la diferencia?

Carlos Iberico, Debora Vansteenwegen, Bram Vervliet y Dirk Hermans. El efecto de la (im)predictabilidad en el miedo contextual: una réplica de hallazgos básicos

Anne Marie Costalat-Founeau. Dinámica de la identidad, acción y contexto 
Revista de Psicología Vol. XXV (1), 2007 (ISSN 0254-9247)

\title{
Dinámica de la identidad, acción y contexto
}

\author{
Anne Marie Costalat-Founeau ${ }^{1}$ \\ Universidad Paul Valéry Montpellier 3
}

\begin{abstract}
La identidad social no puede ser considerada más como una imagen fijada y estática que determina las representaciones individuales y/o colectivas. Se inserta, más bien, dentro de una dinámica de representaciones que está marcada por fases biográficas y sociales. Esta impresión social está en parte construida por la experiencia personal impresa en los recuerdos, y transfiere una realidad experiencial a la identidad. La acción participa en esta concretización y también la refuerza: es así que el carácter normativo de la acción parece reforzar su propia manifestación y, por lo tanto, su efecto. Esos efectos tienen un poder central en la dinámica de la identidad. La acción se ubica en el centro de la subjetividad, pues pone en relación las aspiraciones, las representaciones y también los valores y el sentimiento de capacidad: la acción favorece una relación estrecha entre los factores afectivos y cognitivos. Esos factores actúan en conjunto elaborando regulaciones dinámicas y organizando fases de identidad más o menos conflictivas. Se presentan los efectos de la acción sobre la subjetividad.

Palabras clave: identidad, acción, capacidad, contexto.
\end{abstract}

\section{Identity dynamic, action and context}

Social identity should not be considered anymore as a fixed and static image that defines individual or collective representations. It is embedded within a representational dynamic where biographical and social phases left their marks. This social imprint is partly made by the experience ingrained in the remembrance that gives all its experiential reality to identity. Action participates and reinforces this concreteness. It seems that the prescriptive nature of action reinforces its presence and therefore its effects. More precisely, these effects, which are inherent to action, possess a central power in the identity dynamic. Action is at the heart of subjectivity in the sense that it connects aspirations, representations, as well as the values of the individual and the sense of his own capacity. It involves the tight interweaving of affective and cognitive factors interacting into dynamic regulations and organizing more or less conflicting identity phases. We present in this article the effects of action on subjectivity. Keywords: Identity, action, capacity, context.

1 Doctora en Letras y Ciencias Humanas. Profesora de Psicología Social, responsable del Master en Psicología del Trabajo y las Organizaciones, Universidad Paul Valéry Montpellier 3. Sus intereses de investigación se centran en la identidad social, la acción y el contexto, temática estudiada en el campo de la salud, del trabajo o de las situaciones de transiciones culturales. Dirección postal: Université Paul Valéry Montpellier 3, Route de Mende 34090, Montpellier, Francia. Correo electrónico: Anne-marie.costalat@université.montpellier3.fr 

El objetivo de este artículo es presentar una investigación sobre la cuestión de la representación que el individuo se hace de sí mismo, pero también de la sociedad en relación con la acción y su contexto. Esta cuestión implica necesariamente una dimensión epistemológica en la medida en que posee el estatuto del individuo y el de su relación con el contexto. Por otra parte, este problema ha inspirado varias investigaciones, específicamente las de Carver y Sheier (1985). Se constata que la relación representación-acción es compleja y que parece interesante aprehenderla a partir de ciertos procesos. En este marco, la representación se considera como un mediador importante de integración y socialización, un regulador de la conducta social que orienta al sujeto a través de itinerarios más o menos sinuosos en función de las predisposiciones individuales, interindividuales, contextuales y temporales (Costalat-Founeau, 1997). La representación del sujeto en la acción puede ser considerada como un vector central de la conducta social, en la medida en que ella es preparación y orientación de la acción, y eso atañe a la definición de Moscovici (1976), quien concibe las representaciones sociales como una "preparación de la acción":

El concepto de representación toma sentido gracias a un uso concreto: «La representación social» es una "preparación para la acción», ella no lo es solamente en la medida en que guía el comportamiento, sino también sobre todo en la medida en que ella remodela y reconstruye los elementos del contexto en donde el comportamiento debe tener lugar. Ella viene a dar sentido al comportamiento, a integrarlo en una red de relaciones en donde está vinculado a su objeto, proveyendo al mismo tiempo las nociones, las teorías y el fondo de las observaciones que lo hacen estable y eficaz (p. 46).

Ciertamente, las representaciones son, en principio, puntos de referencia: ellas proveen "una eficacia o una perspectiva" a partir de la cual un individuo o un grupo observa e interpreta los acontecimientos 
y las situaciones. Así, permiten al individuo situarse mejor y ubicar el contexto. Una mejor manera de estudiar la organización de este mundo es a través de la comunicación, el lenguaje y la acción. Se trata entonces de aproximarse a ciertos mecanismos de las representaciones sociales desde el punto de vista del sujeto, tal como él los interioriza, tal como se apropia de ellos, por un conjunto de procesos complejos, en parte ligados a la huella socio-emocional dejada en la vivencia por el contexto experimentado.

Se puede observar que las representaciones sociales que constituyen nuestras ideologías, nuestros conocimientos, no están desprovistas de afectos. Es la influencia del afecto la que da a las representaciones una acción desuniforme, y en este sentido nos remitimos a los trabajos de Zavalloni (1989), para quien la representación social es activada por una red socio-emocional vinculada con la historia social personal. A partir de ahí comprendemos mejor la fuerza y el significado de la acción sobre la dinámica representacional.

Parece que el enfoque de la cuestión por los efectos de capacidad de acción permite integrar una complejidad del problema de forma menos dicotómica y causal, así como evitar la clásica discusión acerca de si la acción precede a la representación o viceversa. A partir de este punto de vista, parece que se puede considerar la cuestión de la representación y de la acción en una causalidad representación-acción y acción-representación, que pone en relación la representación y los efectos de acción que hemos calificado como efecto de capacidad (Costalat-Founeau, 1997). Estas formas de capacidad tienen una influencia importante en la representación del individuo, ya que ellas están directamente ligadas a las movilizaciones socio-emocionales y cognitivas. Esta cuestión entraña la tradicional dificultad que concierne a las modalidades de interacción entre la representación del self — centrada sobre el sujeto- y las representaciones sociales generadas por la sociedad y los grupos. Esta dicotomía tradicional tiende a atenuarse con el enfoque de un individuo complejo, traspasado por representaciones sociales que son compartidas por un 
gran número de individuos pero que son también singulares, ya que ellas están interiorizadas y diferenciadas en función del impacto emocional y cognitivo de la acción que nosotros hemos esquematizado así:

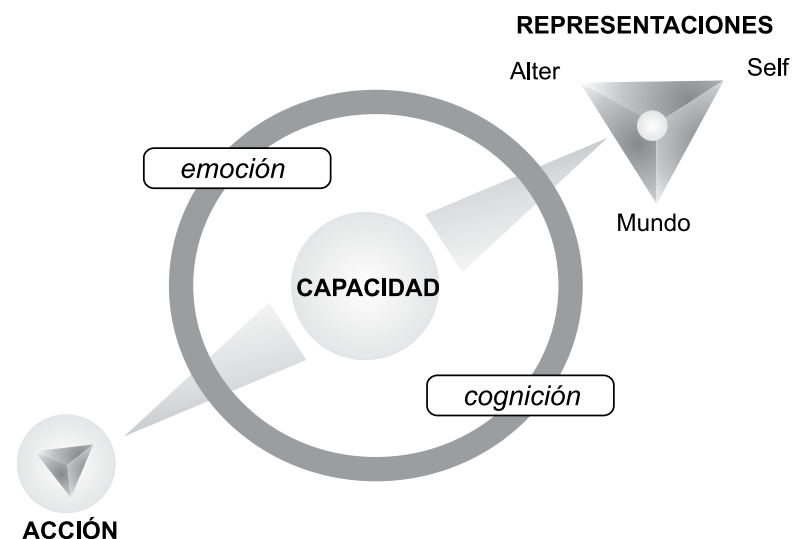

Figura 1. Representación y acción (figura realizada por F. Lepaumier).

\section{Capacidad y acción}

Si examinamos los diferentes significados de la acción, parece que su carácter público refuerza su manifestación y su efecto. Del sentido jurídico al sentido físico, la noción de acción comporta la causa y el efecto. "El orden social se inscribe en el cuerpo a través de esta confrontación permanente, más o menos dramática, pero que deja siempre un gran lugar a la afectividad y, más precisamente, a las transacciones afectivas con el entorno social" (Bourdieu, 1997, p. 169).

Acción significa 'actuar sobre', pero también 'ser movido por' (efecto bajo la acción de). En filosofía, acción significa 'ejercicio efectivo de un poder, de una potencia virtual'. La acción es a veces concebida como un verdadero medio, una situación al borde de la acción. En derecho, la palabra sirve para designar la 'posibilidad [que nosotros tenemos] de reclamar justicia'. En términos de comercio, expresa 'la parte de interés que tienen los miembros de ciertas sociedades en los fondos y beneficios de 
esas sociedades'. Este repaso etimológico nos muestra el carácter multidimensional y complejo que se encuentra en psicología. Se puede comparar la acción con una situación comportamental limitada a una situación estructurada que se acerca a la noción del campo de acción de Lewin (1975). Nos podemos centrar sobre su aspecto dinámico, energético. El concepto de acción, a largo plazo, ha sido estudiado a través de la motivación: Nuttin (1980) la califica como un objeto que sostiene la motivación hacia una meta; Ajzen (1985) introduce la dimensión cognitiva de la acción y de su control; Miller (1976), con su modelo, ubica las operaciones sucesivas y su retroalimentación por medio de una estructura jerárquica. No se puede más que lamentar esta organización de la acción. En un enfoque más global, Amerio (1995) la define como una competencia: "La acción introduce en la cognición la dimensión de sí mismo como actor, esta competencia (y el conjunto de esquemas al que está vinculado) puede ser activada más allá de toda conducta efectivamente realizada a nivel de los actos que el individuo sabe o siente posibles o imposibles" (p. 272).

Para Von Cranach (1995), la acción puede ser concebida como una secuencia intencional, y proporciona al individuo un sentido a la vez individual y colectivo. El autor propone un modelo secuencial en co-evolución, que vincula la acción y la representación en una relación compleja que implica procesos a largo plazo y de diferentes niveles. Podemos ver, con Leyens (1983), la consideración de una acción estratégica que pone en escena a un individuo pragmático preocupado por una eficacia inmediata. En sus investigaciones sobre la eficacia del self, Bandura y Schunk (1981) ponen el acento sobre el acceso a los objetivos que proporcionan al individuo satisfacción cuando aquellos están al alcance, y de cierta forma los autores muestran la influencia de la autoestima en la medida en que el niño busca una forma de lograrlos. En una perspectiva de realización de los objetivos y de despliegue de las competencias, una serie de experiencias han mostrado que los niños que presentaban grandes deficiencias en matemáticas y un desinterés marcado por esta materia se han implicado en una serie de sesiones de aprendizaje dirigidas a la meta de alcanzar objetivos accesibles. Los resultados de la 
experiencia confirman el desarrollo de la motivación para actualizar las capacidades por la auto-evaluación de la eficacia personal.

Existe en el individuo una función de autocontrol, por costumbre y por aprendizaje social. Las evaluaciones favorables generan lo que hemos calificado como capacidad normativa positiva y los juicios desfavorables generan una capacidad normativa negativa (Costalat-Founeau, 2001). Por otro lado, se constata que existe en el individuo una reacción autoevaluativa vinculada con una necesidad de integración y de inter-estructuración del contexto (Costalat-Founeau \& Martínez, 2000; Martínez \& Costalat-Founeau, 2005). Cuando los objetivos están situados más allá de las capacidades y no están animados, el individuo define sus propias acciones como forma de ajustar la capacidad subjetiva y la capacidad normativa. Construye entonces un mundo social ajustado a su dimensión, que se convertirá en una situación inter-estructurante. Así, los individuos juzgan verdaderamente sus capacidades a partir de circunstancias, de contextos y de acciones producidas que pueden desarrollarse. La función de control activa su capacidad subjetiva. La evaluación permite al sujeto desarrollarse o no, lo que se puede calificar como realismo socio-cognitivo.

Esta forma de auto-regulación puede, por una parte, promover una acción y, por otra, reforzar la capacidad del individuo a partir de influencias auto-reactivas, conformes con la representación del self que él desea producir (Goffman, 1980). Esta acción debe ser narrada: el otro forma parte de esta historia social.

Harré (1993) concibe la acción como un episodio, una construcción social, que permite la construcción de la identidad a través de un discurso productor de competencias. El individuo es, a la vez, autor, actor y observador en el contexto. Así, ajusta su capacidad subjetiva y objetiva en la relación social integradora. Desde nuestro punto de vista, esta realidad no es estable ni prescrita. El individuo participa en su construcción y maneja el contexto a partir de sus capacidades y con la preocupación permanente de concretizarlas. 
Los efectos que surgen de la acción tienen un lugar mayor y orientan nuestras investigaciones (Costalat-Founeau, 1995a, 1997, 1999, 2001, 2005a, 2005b). Tenemos la hipótesis de que una congruencia entre las dos formas de capacidad (subjetiva y normativa) es necesaria para implicar un proceso de actividad representacional, que se traducirá por una situación de clarividencia favorable a la concepción del proyecto de acción y de su realización.

Este sentimiento positivo de coherencia por la capacidad, hacia el cual el individuo tiende, lo moviliza como actor central en el tratamiento de la información. En psicología cognitiva, se observa este mismo proceso en la memorización. Así, una información es memorizada cuando está en relación con uno mismo en el momento en que se entera. Greenwald (1980) subraya el efecto totalitario del ego. Los trabajos de Bower (1983) van en el mismo sentido: "los sujetos se focalizan sobre su propio comportamiento hasta el punto de retener del comportamiento de otro solamente lo que precede o sigue inmediatamente a su propio comportamiento" (p. 387). Algunas observaciones de la vida cotidiana tienden a mostrarlo: el carácter positivo de las evaluaciones afecta naturalmente la autoestima y mantiene la hipótesis de que el efecto de reconocimiento social tiene un peso manifiesto en la construcción de las representaciones y el tratamiento de la información.

Allport (1943) había avanzado la hipótesis de una interacción estrecha entre la perspectiva por una meta a alcanzar y la movilización del sujeto. Cuando hay un compromiso personal, la personalidad está implicada en los rasgos generales; cuando no hay compromiso personal, ella no está implicada. El contexto representacional puede ser así un factor, un facilitador inhibidor del compromiso en la acción, y eso está en parte vinculado con los efectos de la misma acción. Esta actividad representacional facilitará la producción del contexto simbólico favorable al proyecto de acción. Si se compara este proceso con el de la motivación, es necesario distinguir la noción de acuidad representacional, que es un determinante clave en el compromiso y en la implicación del 
sujeto en la acción, y la movilización energética por alcanzar una meta en la concepción comportamentalista.

Los efectos de capacidad en la acción introducen una dimensión del individuo, a la vez autor de la construcción de su identidad y de su reconocimiento social (Codol, 1979), y tienen una influencia determinante en la realización de la acción. Esta es necesaria para la concretización de las capacidades del sujeto, pero tiene el poder de inducir efectos positivos que entrañan una dinámica representacional del sujeto. Estos últimos activan las tonalidades afectivas que favorecen una forma de clarividencia, una perspicacia para juzgar las cosas con exactitud y un poder de control sobre el contexto o el medio ambiente. Así es como, dentro de una dinámica de capacidad (subjetiva y normativa), se elabora la representación: por la fase de acuidad o difusión. La representación supone, por consiguiente, una dinámica representacional vinculada con las co-regulaciones —emoción y cognición, que producen efectos de acción-. Esta es condicionada por las regulaciones de la acción.

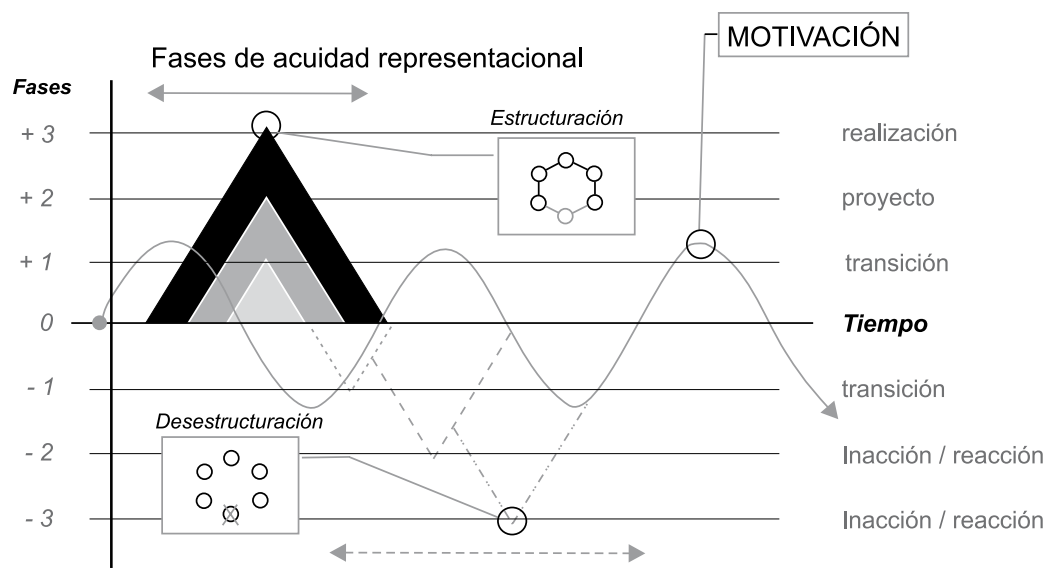

Fases de difusión representacional

Figura 2. Activación de la representación en función de las fases (figura realizada por F. Lepaumier). 


\section{Dinámica representacional y acción}

Nosotros planteamos la hipótesis de que el individuo social tiende hacia una función de concretización de sus capacidades, principalmente por la preocupación de la evaluación social y del reconocimiento social, aunque también para activar su capacidad subjetiva, es decir, su sentimiento de capacidad. Si la acción es una concretización de las capacidades, ella es el medio necesario de regulación de las tonalidades afectivas, movilizadoras de esta misma acción. Ahora desarrollamos la idea de que las dos formas de capacidad no pueden ser consideradas de manera independiente, sino que se activan recíprocamente. Se puede retomar la noción de entrenamiento desarrollada por Piaget y retomada por Huteau (1985) sobre las relaciones entre las dimensiones afectivas y cognitivas, que no implican un vínculo de dependencia pero sí una activación recíproca. Se puede considerar que, para activar una dinámica representacional propicia, la capacidad normativa y la capacidad subjetiva deben ser congruentes. Esto nos conduce a pensar que la movilización emocional ocupa una parte preponderante en la construcción de las representaciones, en estrecha interdependencia con los modos de reconocimiento social. A la vez actor de lo social y director de sus representaciones, el sujeto, en una relación integradora, entabla la acción para producir efectos y eso es un determinante clave de la dinámica representacional.

Este sistema de capacidades se fundamenta en la acción recíproca de dos formas de capacidad interdependientes: la capacidad normativa, directamente vinculada con la validación social, que provee la legitimización social por una forma de validación pública, lo que proporciona reconocimiento social del self, y la capacidad subjetiva, vinculada con la autoestima por uno mismo, de aceptación de uno mismo, manteniendo al mismo tiempo una forma de coherencia interna vinculada con la autoestima.

Este es un vínculo estrecho entre la autoestima y los juicios que otro formula. Si se concede explícitamente una capacidad a una persona, 
esta podrá acrecentar la congruencia entre ese juicio externo y su propia auto-evaluación. Sin embargo, el no reconocimiento social puede entrar en disonancia con la autoestima (situación de incongruencia) y viceversa. El individuo elabora una trayectoria de identidad para encontrar esta coherencia, y tiende hacia un equilibrio de su sistema de capacidad. Sin embargo, las situaciones sociales no siempre facilitan la coherencia. Existen fases difusas (difusión representacional) y fases más claras (acuidad representacional). La fuerza del actor y su poder residen en el ajuste de esas fases más o menos congruentes.

Si este último no accede a un sentimiento de legitimidad, un desorden cognitivo puede desarrollarse; las representaciones se tornarían más inestables y los actos podrían bloquearse. Eso podrá traducirse por un retroceso y por el rechazo de participación que se acercan a las estrategias de coping en su vertiente negativa - retiro, rechazo, enfrentamiento con situaciones estresantes - (Lazarus, Coyne \& Folkman, 1982). Los procesos de coping pueden explicar ciertas actitudes en los sujetos en situación de incertidumbre y particularmente en situación de inestabilidad profesional. Si los fenómenos —en general— son importantes en la construcción de la identidad, los fenómenos de validación social no están vinculados exclusivamente por la institución social. Tenemos el ejemplo de la construcción de identidad de los formadores-capacitadores, actores de la crisis contemporánea y sin estatus oficialmente reconocido, quienes para estar legitimizados construyen espacios de validación en orden a activar su capacidad subjetiva en congruencia con su capacidad normativa y continuar con su acción (Costalat-Founeau, 1995b).

Asimismo, esta condición puede tener como consecuencia la innovación o la construcción de un espacio experiencial informal desconcentrado de su ámbito social institucional. Esta forma de microcosmos es poco representativa de la norma, pero satisface de forma temporal en la medida en que sirve de marco de referencia, de punto de comparación. Se puede evocar el concepto de identidad virtual desarrollado por Dubar (1991) y Sainsaulieu (1988) a propósito de desempleados de la 
formación-capacitación. En su búsqueda de identidad profesional, el individuo persigue y encuentra la validación social, el reconocimiento que facilita una implicación socio-afectiva y que permite elaborar una objetivación construida y limitada para proporcionar una significación a la acción. La capacidad en relación con la acción y su significado pasan por el poder del individuo actor, quien administra y auto-organiza los campos de referencia más o menos objetivados, a fin de permitir una confrontación con el contexto. Esto podría significar que, si el campo de acción no existe o no permite el despliegue de las capacidades, el sujeto lo construye y encuentra formas de trampolín de la identidad (Costalat-Founeau, 1995b). La acción se convierte en una regulación operacional de la representación.

Tomemos el ejemplo de una formadora-capacitadora que obtiene la valorización social que buscaba. Al principio, sin empleo, ella se convierte en formadora en una asociación; luego es directora de un centro de formación con toda la responsabilidad que este tipo de puesto implica. Sin embargo, confiesa que no es posible representarse a sí misma y desarrolla un sentimiento negativo de sí misma: se constituye, pues, como una prisionera de su legitimidad social. Eso entraña una forma de disonancia entre el rol que ella ocupa, los valores que ella defiende y su capacidad subjetiva. Este encuentro fue un punto importante en nuestra investigación y nos condujo a ir más lejos en la elaboración de un modelo capacitario, fundado sobre procesos de congruencia indispensables para el desarrollo de una buena representación de uno mismo.

Observamos que los procesos de identidad complejos dependen de la congruencia de la capacidad normativa y subjetiva. Así pues, no se puede considerar el rol y el estatus vinculados con la capacidad normativa como los únicos determinantes de la dinámica. Existe una capacidad subjetiva en relación con la autoestima y las tonalidades afectivas que puede colorear la representación, y es en una interacción constante como debemos considerar el problema. Representarse se convierte, entonces, en tener simultáneamente una forma de clarividencia, es decir, 
una acuidad representacional fina con una definición de la imagen de uno mismo y la capacidad para administrar su conducta, sus límites, sus medios, lo que es necesario para concretizarla.

En la dinámica representacional, el significado de la noción de capacidad tiene un sentido fuerte, como lo dice Ricoeur (2004). Es en la realización y en la actualización de esta capacidad que se generan las competencias, lo cual facilita la concretización de un efecto de capacidad multifuncional. En efecto, no se trata de una competencia asociada a la realización de una tarea específica: se trata de una dinámica social compleja que hemos ilustrado particularmente con la experiencia de los formadores-capacitadores (Costalat-Founeau, 2005). En efecto, para un grupo en vías de profesionalización — como es el caso de estos formadores - se han puesto en evidencia los mecanismos de construcción de la identidad profesional en el mismo instante de la gestión de los grupos de formación.

Estos autores sociales (Costalat-Founeau, 1999) construyen una identidad en una sociedad en crisis al desplegar, en congruencia, su capacidad subjetiva y normativa. En el corazón de la crisis, estos formadores encuentran respuestas que son las suyas. Despliegan entonces, en una congruencia excepcionalmente eficaz, sus valores y sus capacidades, y los anclan en una realidad en crisis que ellos mismos generan. Se puede constatar el poder de la acción sostenida por un deseo de actualización de las capacidades. Teniendo en cuenta la importancia de los valores que ellos desean conservar, se podría evocar la noción de ideología operacional que los mantiene en esta búsqueda representacional.

\section{Conclusión}

La representación no puede ser estudiada fuera de una dinámica activada por factores socio-emocionales, por una parte, y cognitivos, por otra, y esto en estrecha interacción con el contexto que incluye espacios 
temporales y sociales. Esta dinámica representacional está regulada por la acción que se convierte en un vector central en la representación y que permite formas de objetivación social, aunque también experienciales y axiológicas. Parece importante integrar esta noción de variabilidad vinculada con la temporalidad y con el contexto, ya que la acción se desarrolla en una trayectoria de vida como una obra de teatro se desarrolla en varios actos (Goffman, 1980). Sin embargo, para comprometerse en la acción, recordamos que la representación de uno mismo y del contexto necesita una forma de clarividencia del individuo. Esta actitud sobresale particularmente durante la investigación de un nuevo proyecto de vida o de la puesta en marcha del proyecto profesional. Sin embargo, el sujeto puede elegir entre no reaccionar y quedarse en una representación más difusa. Difusión y acuidad representacional, así, pueden alternarse. La acción es un evento secuencial; se convierte en un medio de regulación de los efectos de capacidad, pero esos mismos efectos no son constantes, ya que existen momentos de reflexión. El sujeto no está siempre en acción: puede retroceder:

Ciertas personas se retiran físicamente por largos periodos; retroceden para reflexionar sobre sus problemas de identidad y regresan con respuestas sobre el plan social o profesional. Nosotros lo hacemos a una mínima escala cuando nos concedemos momentos de intimidad o de recogimiento. En cambio, hay fases esenciales de la vida que implican virajes y contactos sociales apasionantes, que permiten prolongar la comunicación, cuando, por ejemplo, se siente la necesidad de reforzar rápidamente y enérgicamente una imagen de sí mismo que vacila, la de validar y revalidar una concepción de sí mismo recientemente descubierta. Es posible que los períodos de rápida adquisición de conocimientos estén estrechamente ligados a esas fases (Strauss, 1992, p. 137).

Esta tensión de congruencia es especialmente activada para poner en marcha, por ejemplo, una identidad profesional, pero no está siempre al máximo (Guillen \& Costalat-Founeau, 2002). Como subraya Lemaine (1974), el individuo se desplaza a los lugares donde puede producir y construir una identidad social coherente con sus valores. Es en una 
dinámica constante que el sujeto organiza sus capacidades. Sin embargo, como hemos evocado, los intereses pueden ser modificados, pues varían según las fases. Tomar en consideración la acuidad o la difusión representacional del sujeto es subrayar la importancia de la variabilidad condicionada por la intensidad de la fase de vida en función de la temporalidad de los objetivos y de los proyectos. Los efectos de capacidad de la acción son una variable importante que se debe tomar en consideración. Para reaccionar, es necesario ser capaz de representarse bien a sí mismo, pero también al contexto o medio ambiente, y la acción sirve como medio de construcción para esta misma representación.

La acción interviene como una forma de regulación operacional de la representación; puede ser considerada como un campo experiencial y temporal necesario para activar la dinámica representacional. Existe, al parecer, un significado a la vez socio-afectivo y socio-cognitivo de la acción. Esta deviene de los efectos de capacidad, manifestación, transformación y concretización de las representaciones. A partir de ahí se puede tomar el poder del actor dentro de su construcción de identidad. La activación de un sentimiento de sí, de una autoestima vinculada con la legitimidad social en coherencia con los valores del sujeto, será caracterizada como una consonancia axiológica. Esta es una condición fundamental para elaborar proyectos de acción y para comprometer las estrategias. Las capacidades inherentes al desarrollo de la acción se convierten, así, en poderosas reguladoras de las estrategias de la capacidad del individuo y de sus representaciones. La acción es también el medio necesario de regulación de tonalidades afectivas, movilizadoras de ella misma, así como el vínculo entre un dominio intrínseco del individuo y el contexto social.

Ahora bien, la dimensión socio-emocional tiene una influencia mayor en la construcción de las representaciones, en la que la influencia de los valores debe ser subrayada. El carácter a la vez axiológico (valores), histórico (biográfico), simbólico (significación) y legítimo (poder) de la acción monitorea la expresión del sujeto social, su traza histórica y cultural. 
Si intentamos ubicar el trabajo del individuo, le otorgamos cuatro tipos de poder: uno normativo (legitimidad social), uno socio-cognitivo (activación de los conocimientos), otro socio-emocional (en vinculación con las tonalidades afectivas y la autoestima) y uno axiológico, que comprende los valores (Costalat-Founeau, 2001). Definiendo la acción como una regulación operacional de las representaciones del individuo, hacemos alusión, de cierta forma, a Von Cranach (1995), quien concede a la acción un poder de transformación de las representaciones sociales en representaciones sociales individuales, panorama en el que la acción cumple una función de transformación. Pero para que este proceso sea eficaz, es necesario, según parece, que la capacidad subjetiva y la capacidad normativa estén en congruencia.

Si nos centramos en el individuo en situación de representación dentro de la acción, así como en las capacidades de las que él dispone a nivel intra-individual, inter-individual y contextual, la cuestión se enfoca según el proceso que es necesario explorar aún. Si la acción puede ser definida en forma de secuencia como un proyecto en temporalidad mediana, nos parece que el vivo debate que se opone al lugar que ocupa la acción dentro de las representaciones podría esclarecerse en los efectos de capacidad de acción en vinculación con la red activadora de las capacidades socio subjetivas y normativas.

\section{Referencias}

Ajzen, I. (1985). From intention to actions: A theory of planned behavior. En J. Kuhl \& J. Beckmann (Eds.), Action control: From cognition to behavior (pp. 11-39). Berlin: Springer-Verlag.

Allport, G. W. (1943). The ego in contemporary psychology. Psychological Review, 50, 451-478.

Amerio, P. (1995). Fondamenti thorici di psicologia sociale. Bologna: Il Mulino. 
Bandura, A. \& Schunk, D. H. (1981). Cultivating competence, selfefficacy and intrinsic interest through proximal self-motivation. Journal of Personality and Social Psychology, 41(3), 586-598.

Bourdieu, P. (1997). Méditations pascaliennes. Paris: Seuil.

Bower, G. H. (1983). Affect and cognition. Philosophical Transactions of the Royal Society of London, 302(b), 387-402.

Carver, C. S. \& Sheier, M. F. (1985). A control-systems approach to the self-regulation of action. En J. Kuhl \& J. Beckmann (Eds.), Action control: From cognition to behavior (pp. 237-265). Nueva York: Springer-Verlag.

Codol, J. P. (1979). Semblables et différents. Recherches sur la quête de la similitude et de la différenciation sociale. Tesis no publicada, Université de Provence, Aix-en-Provence, Francia.

Costalat-Founeau, A. M. (1995a). La representation professionnelle du formateur : Le lien crisique, le lien tremplin, le lien scénique. L'Orientation Scolaire et Professionnelle, 24(3), 273-299.

Costalat-Founeau, A. M. (1995b). Representations, representation de soi, une question épistémologique. Papers on Social Representations, Threads of Discussions, 4(1), 618-622.

Costalat-Founeau, A. M. (1997). Identité sociale et dynamique representationnelle. Rennes, Francia: Presses Universitaires de Rennes.

Costalat-Founeau, A. M. (1999). Identity dynamics, action and context. Journal for the Theory of Social Behaviour, 29(3), 289-300.

Costalat-Founeau, A. M. (2001). Identité, capacité et langage. En A. M. Costalat-Founeau (Ed.), Identité sociale et langage: La construction du sens. Paris: L'Harmattan.

Costalat-Founeau, A. M. (2005a). (Ed.) Identité sociale et ego-écologie, théorie et pratique. Paris: Sides.

Costalat-Founeau, A. M. (2005b). Identité, action et representations sociales. En A. M. Costalat-Founeau (Ed.), Identité sociale et egoécologie: Théorie et pratique (pp. 69-101). Paris: Sides.

Costalat-Founeau, A. M. \& Martínez, N. (2000). Identité sociale et réussite: Comparaison interculturelle qualitative selon l'approche 
ego-écologique. Cahiers Internationaux de Psychologie Sociale, 47-48, 165-194.

Dubar, C. (1991). La socialisation: Construction des identités sociales et professionnelles. Paris: Armand Colin.

Goffman, E. (1980). La mise en scène de la vie quotidienne. La presentation de soi. Paris: Minuit.

Greenwald, A. G. (1980). The totalitarian ego: Fabrication and revision of personal history. American Psychologist, 35, 603-618.

Guillen, S. \& Costalat-Founeau, A. M. (2002). Identité et changement professionnel. Le cas des infirmières faisant fonction de cadre. Psychologie du Travail et des Organisations, 8(2), 177-198.

Harré, R. (1993). Social being (2da. ed.). Oxford: Blackwell.

Huteau, M. (1985). Les conceptions cognitives de la personnalité. Paris: Presses Universitaires de France.

Lazarus, R. S., Coyne, J. C. \& Folkman, S. (1982). Cognition emotion and motivation: The doctoring of Humpty-Dumpty. En R. W. J. Neufeld (Ed.), Psychological stress and psychopathology. Nueva York: McGraw-Hill.

Lemaine, G. (1974). Social differentiation and social originality. European Journal of Social Psychology, 4, 17-52.

Lewin, K. (1975). Psychologie dynamique: Les relations humaines (5a. ed.). Paris: Presses Universitaires de France.

Leyens, J. P. (1983). Qu'est-ce qu'un bon jugement social. En J. L. Beauvois, R. V. Joule \& J. M. Monteil (Eds.), Perspectives cognitives et conduites sociales: Jugements sociaux et changements des attitudes (pp. 71-81). Neuchatel: Delachaux et Niestlé.

Martínez, N. \& Costalat-Founeau, A. M. (2005). Comparaison de deux situations de réussite confrontée et normalisée chez des lycéennes d'origine culturelle différente. Connexions, 83, 149-168.

Miller, D. T. (1976). Ego-involvement and attribution for success and failure. Journal of Personality and Social Psychology, 34, 901-906.

Moscovici, S. (1976). La psychanalyse, son image et son public. Paris: Presses Universitaires de France. 
Nuttin, J. (1980). Motivation et perspectives d'avenir. Lovaina, Bélgica: Presses Universitaires de Louvain.

Ricoeur, P. (2004). Parcours de la reconnaissance. Paris: Stock.

Sainsaulieu, R. (1998). L'identité au travail d'hier à aujourd'hui. L'Orientation Scolaire et Professionnelle, 27, 77-93.

Strauss, A. (1992). Miroirs et masques. Une introduction à linteractionnisme. Paris: Métaillé.

Von Cranach, M. (1995). Individual actions: Misunderstanding omissions and different premises. Journal for the Theory of Social Behaviour, 24, 285-293.

Zavalloni, M. (1989). L'effet de résonance dans la création de l'identité et des representations sociales. Revue Internationale de Psychologie Sociale, 3(3), 407-408.

Recibido 29 de enero, 2007 Aceptado 30 de julio, 2007 